\title{
ChemComm
}

\section{Polynorbornene derived 8-hydroxyquinoline paper strips for ultrasensitive chemical nerve agent surrogate sensing $\dagger$}

50, 8511

Received 5th May 2014,

Accepted 5th June 2014

DOI: $10.1039 / c 4 c c 03361 b$

www.rsc.org/chemcomm

The detection of nerve agent simulants is achieved by the photoinduced electron transfer (PET) mechanism. A "turn-on" fluorescence response upon phosphorylation at 8-hydroxyquinoline of norbornene-based triazolyl functionalized 8-hydroxyquinoline (NCHQ) followed by intramolecular rearrangement provides very intense green emission. The detection limit of polymer (PNCHQ) coated paper strips is 25 ppb with instantaneous response.

Chemical warfare agents are produced not only to kill people in war, but also to terrorize people even in peace. ${ }^{1 a}$ Among the nerve gas agent family, sarin is the most dangerous and frequently used gas for the attack. ${ }^{1 b}$ It is well known that nerve gases act as scavengers of acetylcholinesterase, inhibiting its reactivity in the nervous system, which leads to several neurological disorders and even death. ${ }^{2}$ Existing techniques, such as gas chromatography-mass spectrometry, ${ }^{3}$ surface acoustic wave (SAW) devices, ${ }^{4}$ molecular imprinting, ${ }^{5}$ flame photometric detectors ${ }^{6}$ etc., are facing operational complexity, high cost and require a long detection time. So there is always a need for a portable and facile sensing system. Nerve agent simulants, such as diethyl chlorophosphate (DCP), diisopropyl fluorophosphate (DFP), diphenyl chlorophosphate (DPCP), are explored in the literature as they are less toxic. ${ }^{7}$ Swager $e t$ al. developed a unique sensor system that cyclises in the presence of nerve gases and produces fluorescence response. ${ }^{8}$ Based upon photoinduced electron transfer (PET), Rebek et al. have demonstrated another facile technique to detect nerve gases. ${ }^{9}$ There are also reports on using supernucleophiles for nerve gas sensing by Anslyn $e t$ al. ${ }^{10}$ Even various lanthanide complexes were explored as fluorometric sensor systems for nerve agent surrogates. ${ }^{11}$ But most of the existing methods of sensing are either in very high polar solvents like DMF and DMSO or they require alkaline conditions. ${ }^{12}$ According to Centres for Disease Control and Prevention (CDC), the concentration of sarin that is immediately dangerous to life is found to be $0.1 \mathrm{mg} \mathrm{m}^{-3}$

Polymer Research Centre, Department of Chemical Sciences, Indian Institute of Science Education and Research Kolkata (IISER K), India. E-mail: sraja@iiserkol.ac $\dagger$ Electronic supplementary information (ESI) available: Detailed synthesis, characterization, and selected UV and fluorescence data. See DOI: 10.1039/c4cc03361b
(1.7 ppm vapour). So there is always a need for a very sensitive system which can be used as an 'in-field' detector.

Though many existing nerve agent sensors have been reported in the literature, polymer based sensors for 'in-field' application have not been explored so far. Here we report a polymer based "turn-on" fluorometric sensing approach using a norbornene derived 8-hydroxyquinoline motif. 8-Hydroxyquinoline (8-HQ) acts as a weak fluorophore ${ }^{13}$ due to excited state intramolecular proton transfer (ESIPT) from oxygen to nitrogen. ${ }^{14}$ When 8 -HQ is attached to the norbornene functionality by click chemistry, a triazole functionality is generated. Photoinduced electron transfer (PET) from the triazole nitrogen to $8-\mathrm{HQ}$ is the reason for the nonemissive nature of NCHQ. As the non-bonding electrons are involved in phosphorylation, it is obvious that they are not available for the PET process. Due to this a turn-on response is achieved. The sensing response of NCHQ and its polymer, PNCHQ, to the simulant of a nerve agent surrogate is highly sensitive and selective. To the best of our knowledge, this is the first report on a polymeric sensor that has instantaneous 'turn-on' response and a ppb level detection limit upon exposure to the nerve agent simulants.

First of all, 8-HQ was functionalized with alkyne to generate compound 1 (Scheme 1). 11-Bromoundecanol was converted to 11-azidoundecanol (2). $\mathrm{Cu}(\mathrm{I})$ catalyzed 1,3 dipolar cycloaddition of 1 and 2 yielded compound 3 and it was confirmed through the characteristic proton signal at $7.7 \mathrm{ppm}$ by ${ }^{1} \mathrm{H}$ NMR spectroscopy (Fig. S3, ESI $\dagger$ ). ESI-mass and ${ }^{13} \mathrm{C}$ NMR further confirmed the formation of a pure product (Fig. S4 and S8, ESI $\dagger$ ). Finally, norbornene acid was coupled with 3 using the DCC coupling reagent to produce monomer $\mathbf{N C H Q}$. It must be noted that the norbornene was attached to the 8-HQ moiety through a long alkyl chain to avoid solubility issues. The successful formation of NCHQ was confirmed by ${ }^{1} \mathrm{H},{ }^{13} \mathrm{C}$ NMR and mass spectroscopic techniques (Fig. S5, S7 and S9, ESI†). Ring opening metathesis polymerization (ROMP) using Grubbs' second generation catalyst was employed to polymerize NCHQ due to its functional group tolerance. ${ }^{15}$ Formation of a polymer (PNCHQ) was confirmed by the absence of the norbornene double bond peak at $6.1 \mathrm{ppm}$ and the appearance of a new peak at 5.5-5.6 ppm (Fig. S6, ESI†). The molecular weight 


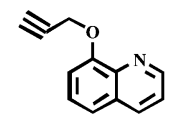

1

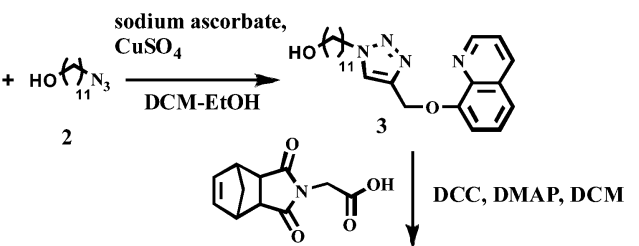<smiles>[Mg][Mg]</smiles>



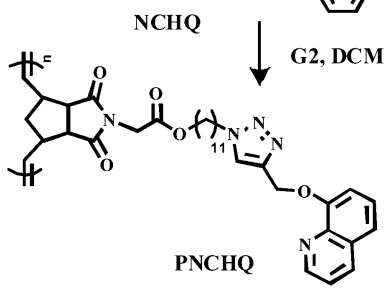

Scheme 1 Schematic representation of synthesis of NCHQ and PNCHQ

and PDI of the polymer were obtained by GPC analysis. The detailed synthesis procedure with characterisation is given in the ESI. $\dagger$

After the successful synthesis of NCHQ and PNCHQ, their ability to detect nerve agent surrogates was explored. First, the response of NCHQ in methanol against the nerve agent surrogate, DCP, was monitored through UV and fluorescence spectroscopy. NCHQ $(3.33 \mu \mathrm{M})$ showed three absorption maximum bands at 202, 240 and $310 \mathrm{~nm}$ in UV spectra. Upon addition of DCP solution, the absorbance bands at $240 \mathrm{~nm}$ and $310 \mathrm{~nm}$ were observed to undergo a red shift towards $250 \mathrm{~nm}$ and $340 \mathrm{~nm}$, respectively (Fig. 1a).

Interestingly, a red shift of $90 \mathrm{~nm}$ was observed upon addition of DCP to NCHQ $(3.33 \mu \mathrm{M})$ solution by fluorescence spectroscopy. Initially NCHQ emission peak was observed at $398 \mathrm{~nm}$ upon excitation at $300 \mathrm{~nm}$. In the presence of DCP, a new emission band at $488 \mathrm{~nm}$ was found upon excitation at $300 \mathrm{~nm}$ (Fig. 1b). A ratiometric emission spectrum was obtained upon gradual addition of DCP to NCHQ, where emission intensity at $398 \mathrm{~nm}$ decreased while emission intensity at $488 \mathrm{~nm}$ gradually increased (Fig. S10, ESI $\dagger$ ). An isoemissive point was found at $450 \mathrm{~nm}$. The lowest detection limit was found to be $25 \mathrm{ppb}$ of DCP in methanol (Fig. S11, ESI $\dagger$ ). Quantum yield of NCHQ was calculated to be 0.053 taking quinine sulphate as a standard. After the addition of DCP to NCHQ, a huge increase in quantum yield (0.146) was observed. This supported the strong 'turn-on' emission response upon addition of DCP to NCHQ. The response of NCHQ was also studied under a handheld UV-lamp to demonstrate naked eye detection. NCHQ in methanol was colourless but instantaneous green emission was observed upon addition of DCP under UV light. Selectivity of NCHQ towards DCP was confirmed when no change in emission was observed with addition of other phosphate reagents (Fig. S12, ESI†). Response of NCHQ against $\mathrm{HCl}$ solution was tested and compared with the response against DCP. The emission response of $\mathrm{HCl}$ was found to be very less compared to DCP (Fig. S14, ESI†). The ability of the sensor molecule NCHQ to detect other organophosphate nerve agent surrogates was also tested. Diphenyl chlorophosphate (DPCP) was added to the solution of NCHQ in
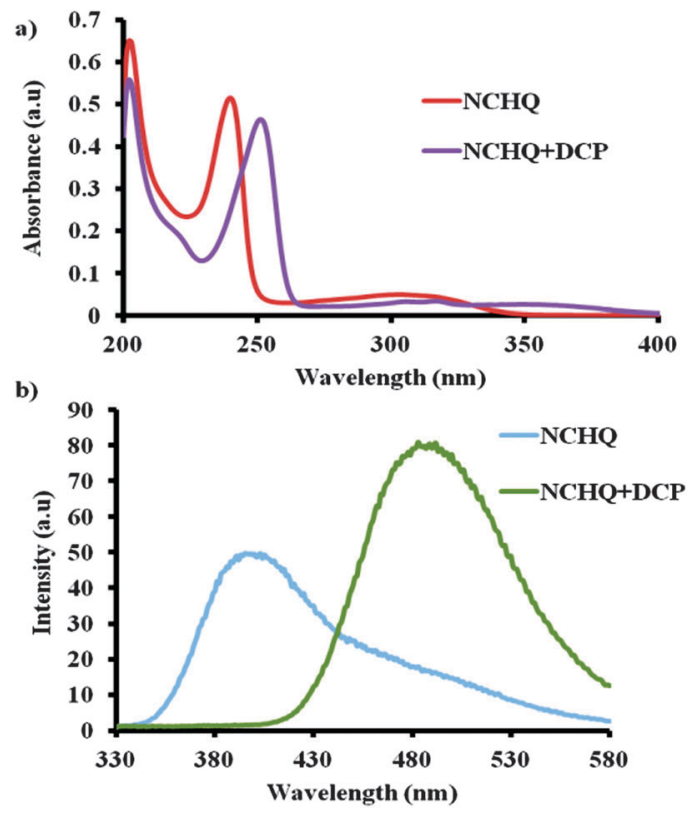

Fig. 1 (a) UV-vis absorption spectra and (b) emission spectra of $3.33 \mu \mathrm{M}$ NCHQ before and after the addition of DCP in methanol.

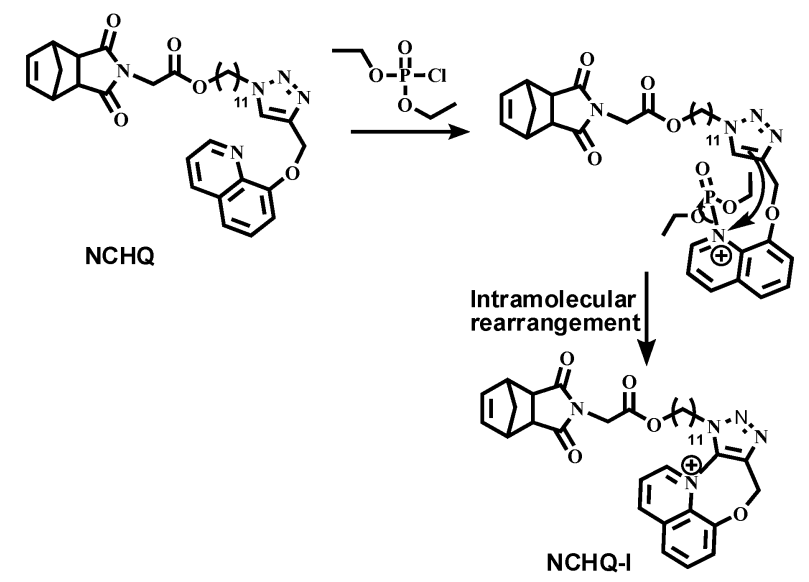

Scheme 2 A schematic proposal for the intramolecular rearrangement.

methanol and the emission spectrum was recorded by exciting at $300 \mathrm{~nm}$. The same spectral shift of $90 \mathrm{~nm}$ with enhancement in the intensity was observed as with DCP (Fig. S15, ESI†). This control experiment prompted us to propose the phosphorylation mechanism for the sensing event (Scheme 2).

To explore the unusual red shift, the mechanism of the phosphorylation reaction between NCHQ and DCP was investigated. ${ }^{1} \mathrm{H}$ NMR spectra were recorded for NCHQ and NCHQ with DCP in $\mathrm{CDCl}_{3}$. It was found that NCHQ aromatic protons, responsible for 8-HQ, shifted to the downfield region upon addition of DCP (Fig. S13, ESI $\dagger$ ). 8-HQ protons in NCHQ appeared at $\delta$ 8.9, 8.2, 7.5, 7.4 and $7.3 \mathrm{ppm}$. Upon addition of DCP, a shift in all the peaks (at $\delta$ 9.3, 8.9, 8.0, 7.9 and $7.8 \mathrm{ppm}$ ) was observed as shown in Fig. 2. Also the characteristic proton signal at $7.7 \mathrm{ppm}$ for the triazole double bond was absent after DCP addition. The shift in the signals was attributed to the nucleophilic attack of $8-\mathrm{HQ}$ nitrogen to the 




Fig. 2 Peak shifting in ${ }^{1} \mathrm{H}$ NMR spectra of NCHQ (a) before and (b) after the addition of DCP.

electrophilic centre of the organophosphate. First, it was assumed to be a NCHQ-DCP complex. But the ESI-MS experiment did not support the proposal. Pure NCHQ displayed a mass peak at 600 . Upon addition of DCP to NCHQ, a peak of the complex was expected to occur in the mass spectrum. Surprisingly, it still showed an intense 600 peak which prompted us to explore the other possible mechanisms. To confirm the formation of new molecules, TLC analysis was performed. Interestingly, a fluorescent spot with higher polarity was observed. Based on mass and TLC analyses, we proposed that two molecules with the same mass but different polarities could be possible only when there was an intramolecular rearrangement. This prompted us to hypothesise that the double bond of triazole was involved in the cyclisation process to form NCHQ-I. Our proposed structure along with the mechanism are given in Scheme 2.

To further confirm the formation of NCHQ-I, NCHQ was reacted with the well-known alkylating agent tosyl chloride and changes were monitored through fluorescence, mass and NMR spectroscopy. It was observed that in the emission spectra tosyl chloride induces the same $90 \mathrm{~nm}$ red shift, as DCP and DPCP, when added to NCHQ solution (Fig. S16, ESI $\dagger$ ). As expected, ESI-MS analysis of NCHQ-TsCl and NCHQ-DPCP produced the characteristic mass peak at 600.53, due to the formation of NCHQ-I through our proposed intramolecular rearrangement (Fig. S17 and S18, ESI $\dagger$ ). The ${ }^{1} \mathrm{H}$ NMR spectral shift of NCHQ-TsCl was found to be the same as NCHQ-DCP (Fig. S19, ESI $\dagger$ ).

After demonstrating the sensing ability of NCHQ successfully, the detection ability of its polymer, PNCHQ, was explored through fluorescence spectroscopy (Fig. S21, ESI $\dagger$ ). Reactivity of PNCHQ towards DCP was monitored through ${ }^{1} \mathrm{H}$ NMR analysis and the corresponding downfield shifts in the peak positions were observed (Fig. S20, ESI $\dagger$ ). Paper strips were made from solutions of PNCHQ $\left(0.5 \mathrm{mg} \mathrm{ml}^{-1}\right)$. A strip of Whatman filter paper was coated with PNCHQ solution and dried. The strip was kept hanging from the inside wall of a glass chamber so that it remained above the bottom level. Under the UV light, the PNCHQ coated paper strip was colourless. Interestingly, when a few drops of DCP were added into the glass chamber, an immediate 'turn-on' response was observed (video attached). It was even seen that holding the strip over the DCP solution also produced a colour change of the strip as shown in Fig. 3. The responsive nature of the PNCHQ coated strip towards DCP in the presence of other chlorinated compounds and water vapour was observed to remain undisturbed (Fig. S22 and S23, ESI $\dagger$ ). These experiments demonstrated the ultrasensitivity of our new polymeric nerve agent sensor.
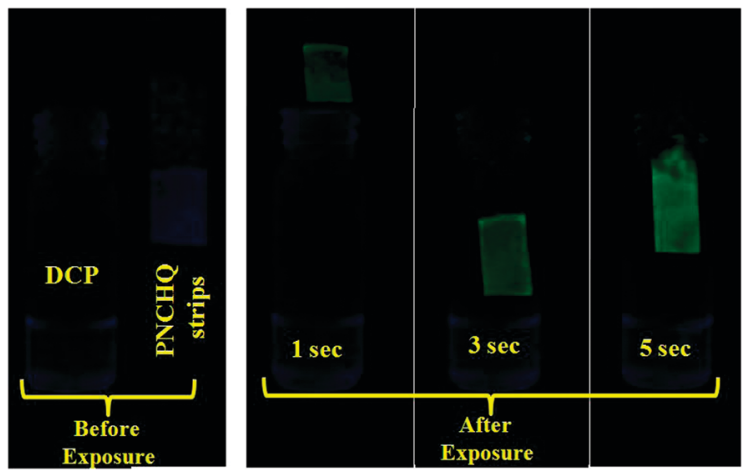

Fig. 3 Demonstration of vapour phase sensing of DCP using a PNCHQ coated paper strip under a UV lamp.

In conclusion, we have demonstrated the ultrasensitive detection of a nerve agent surrogate, DCP, using NCHQ and PNCHQ sensors. From NMR and mass analysis, an intramolecular rearrangement has been proposed for the unique 'turn-on' response. Paper strips of PNCHQ show an instantaneous response upon exposure to DCP. Most interestingly, the paper strips enable the possibility of vapour phase sensing of DCP at a concentration as low as $25 \mathrm{ppb}$, and hence can be utilized for in-field applications.

\section{Notes and references}

1 (a) Y. C. Yang, J. A. Baker and J. R. Ward, Chem. Rev., 1992, 92, 1729; (b) A. T. Tu, Toxin Rev., 2007, 26, 231.

2 (a) C. H. Gunderson, C. R. Lehmann, F. R. Sidell and B. Jabbari, Neurology, 1992, 42, 946; (b) F. R. Sidell and J. Borak, Ann. Emerg. Med., 1992, 21, 865.

3 W. E. Steiner, S. J. Klopsch, W. A. English, B. H. Clowers and H. H. Hill, Anal. Chem., 2005, 77, 4792.

4 (a) Y. Yang, H.-F. Hi and T. Thundat, J. Am. Chem. Soc., 2003, 125, 1124; (b) C. Hartmann-Thompson, J. Hu, S. N. Kaganove, S. E. Keinath, D. L. Keeley and P. R. Dvornic, Chem. Mater., 2004, 16, 5357.

5 A. L. Jenkins and M. O. Uy, Anal. Commun., 1997, 34, 221.

6 S. Kendler, A. Zaltsman and G. Frishman, Instrum. Sci. Technol., 2003, 31, 357.

7 (a) S. Royo, R. Martinez-Manez, F. Sancenon, A. M. Costero, M. Parra and S. Gil, Chem. Commun., 2007, 4839; (b) L. Ordronneau, A. Carella, M. Pohanka and J.-P. Simonato, Chem. Commun., 2013, 49, 8946; (c) L. Feng, C. J. Musto, J. W. Kemling, S. H. Lim and K. S. Suslick, Chem. Commun., 2010, 46, 2037; (d) A. M. Costero, M. Parra, S. Gil, R. Gotor, R. Martínez-Mañez, F. Sancenón and S. Royo, Eur. J. Org. Chem., 2012, 4937; (e) G. H. Dennison, M. R. Sambrook and M. R. Johnston, Chem. Commun., 2014, 50, 195. 8 S.-W. Zhang and T. M. Swager, J. Am. Chem. Soc., 2003, 125, 3420.

9 T. J. Dale and J. Rebek Jr., J. Am. Chem. Soc., 2006, 128, 4500.

10 (a) K. J. Wallace, J. Morey, V. M. Lynch and E. V. Anslyn, New J. Chem., 2005, 29, 1469; (b) K. J. Wallace, R. I. Fagbemi, F. J. Folmer-Andersen, J. Morey, V. M. Lynth and E. V. Anslyn, Chem. Commun., 2006, 3886.

11 (a) R. Shunmugam and G. N. Tew, Chem. - Eur. J., 2008, 14, 5409; (b) S. Sarkar, A. Mondal, A. K. Tiwari and R. Shunmugam, Chem. Commun., 2012, 48, 4223.

12 (a) S. Han, Z. Xue, Z. Wang and T. B. Wen, Chem. Commun., 2010, 46, 8413; (b) A. M. Costero, S. Gil, M. Parra, P. M. E. Mancini, R. MartinezManez, F. Sancenon and S. Royo, Chem. Commun., 2008, 6002.

13 S. Sarkar and R. Shunmugam, ACS Appl. Mater. Interfaces, 2013, 5, 7379.

14 E. Hao, T. Meng, M. Zhang, W. Pang, Y. Zhou and L. Jiao, J. Phys. Chem. A, 2011, 115, 8234.

15 (a) R. H. Grubbs, Handbook of Metathesis, Wiley-VCH, New York, 2003, vol. 3; (b) S. Bhattacharya, S. Sarkar and R. Shunmugam, J. Mater. Chem. A, 2013, 1, 8398; (c) S. Bhattacharya, V. N. Rao, S. Sarkar and R. Shunmugam, Nanoscale, 2012, 4, 6962. 